\title{
An Incidental Chorda Tympani Schwannoma Identified During Middle Ear Surgery
}

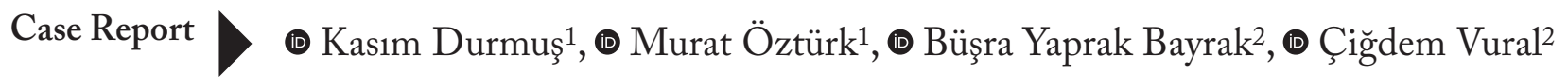 \\ ${ }^{1}$ Department of Otorhinolaryngology, Head and Neck Surgery, Kocaeli University Faculty of Medicine, Kocaeli, Turkey \\ ${ }^{2}$ Department of Pathology, Kocaeli University Faculty of Medicine, Kocaeli, Turkey
}

\begin{abstract}
This study was presented as a poster at $39^{\text {th }}$ Turkish National Otorhinolaryngology, Head and Neck Surgery Congress on November 8th12th, 2017, Antalya, Turkey.
\end{abstract}

\section{ORCID ID of the authors:}

K.D. 0000-0003-1013-067X; M.Ö. 0000-0002-3340-9975; B.Y.B. 0000-0002-0537-3127; C.V. 0000-0002-9405-9112.

Cite this article as: Durmuş $K$, Öztürk $M$, Yaprak Bayrak B, Vural Ç. An Incidental Chorda Tympani Schwannoma Identified During Middle Ear Surgery. Turk Arch Otorhinolaryngol 2021; 59(3): 234-8.

Corresponding Author: Kasım Durmuş; kasimdurmus@yahoo.com

Received Date: 05.05.2021 Accepted Date: 07.06.2021

Content of this journal is licensed under a Creative Commons Attribution 4.0 International License. Available online at www.turkarchotolaryngol.net

\section{(c) (i) $\$$}

DOI: 10.4274/tao.2021-5-2

Chorda tympani schwannomas are rare benign tumors of the middle ear cleft. This is a case of incidental chorda tympani schwannoma identified intraoperatively. The patient was a 50-year-old male with chronic active otitis media complicated by left-sided facial paralysis. During closed mastoidectomy and tympanoplasty approach, a well-demarcated swelling on the left chorda tympani nerve was identified and sectioned for pathologic evaluation. The histopathologic evaluation established the diagnosis of schwannoma. Although the tumor was unlikely the cause of the disease process in this patient, his facial paralysis and middle ear disease were resolved after surgery.

Keywords: Chorda tympani, schwannoma, facial nerve, middle ear tumor, chronic otitis media, case report

\section{Introduction}

Schwannomas are the most common tumors of the facial nerve in the middle ear cleft. They can arise from any segment of the facial nerve or its branches (1). Although isolated chorda tympani schwannomas (CTS) are extremely rare, there are several reported cases in the English literature (2-4). The presented case is an incidental CTS identified during middle ear surgery for chronic otitis media. Clinical and histopathological characteristics of CTS are reviewed in this report.

\section{Case Presentation}

A fifty-year-old male presented to our clinic with a 12-day history of left facial asymmetry. $\mathrm{He}$ had a three-month history of foul-smelling ear discharge. $\mathrm{He}$ used oral ciprofloxacin two times a day for 10 days, topical $0.3 \%$ ciprofloxacin and topical $0.1 \%$ dexamethasone three times a day for three weeks, but his ear discharge persisted. He had no vertiginous symptoms, taste disturbances, or any previous history of facial asymmetry.

On physical examination, he had left-sided House-Brackmann grade 5 peripheral 
facial paralysis. Otomicroscopic examination revealed purulent ear discharge, a bulging mass beneath the posterior quadrant of the tympanic membrane, and a millimetric central perforation just anterior to it (Figures 1a and b). A pure-tone audiogram showed a left-sided conductive hearing loss with an average bone and air conduction thresholds
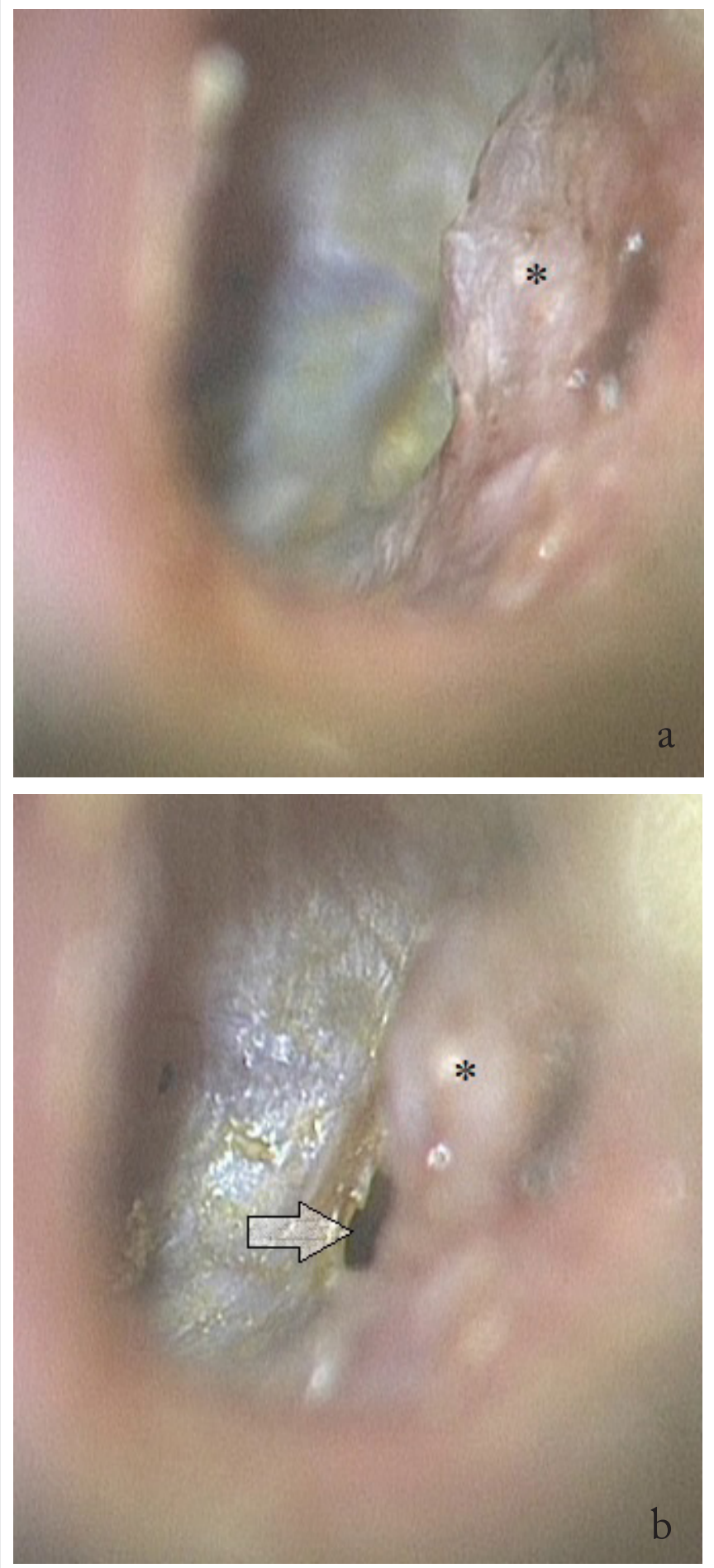

Figure 1. Otomicroscopic view of the patient is showing: (a) a posterosuperior bulge on the left tympanic membrane (asterisk), (b) and a millimetric central perforation (arrow) of $15 \mathrm{~dB}$ and $29 \mathrm{~dB}$ at $500,1,000,2,000$, and $4,000 \mathrm{~Hz}$ frequencies. Electroneurography demonstrated more than 90\% degeneration of the left facial nerve. Temporal bone computed tomography (CT) without contrast showed soft tissue density in the middle ear and mastoid cavity without an identifiable mass lesion. The fallopian canal was intact. Scutum erosion was not present. A closed mastoidectomy was planned to relieve mastoid infection and its burden on the facial nerve.

After elevating the tympanomeatal flap, the surgeon detected that the 1-2 mm segment of the chorda tympani nerve was thickened, which was corresponding to the bulge on the tympanic membrane (Figure 2). The remaining segments of the nerve were normal in size. The fallopian canal was intact. The mastoid cavity was full of inflamed mucosa and exudate, thus mastoidectomy with an intact canal wall was performed. After obtaining verbal consent from the next of kin of the patient, thickened segment of the chorda tympani nerve was excised and sent for histopathologic examination.

Histopathologic evaluation of the specimen demonstrated proliferating spindle cells with a round nucleus, dense chromatin, delicate eosinophilic cytoplasm, and indistinct cell borders in the sections (Figures 3 and 4). There was no cellular atypia, nuclear pleomorphism, or necrotic areas on the specimen. Tumor cells showed diffuse and strong S-100 positivity in the immunohistochemical staining (Figure 5). These histopathological findings confirmed the diagnosis of schwannoma.

During follow-up, the patient's facial paralysis completely resolved two weeks after surgery. Tympanic membrane

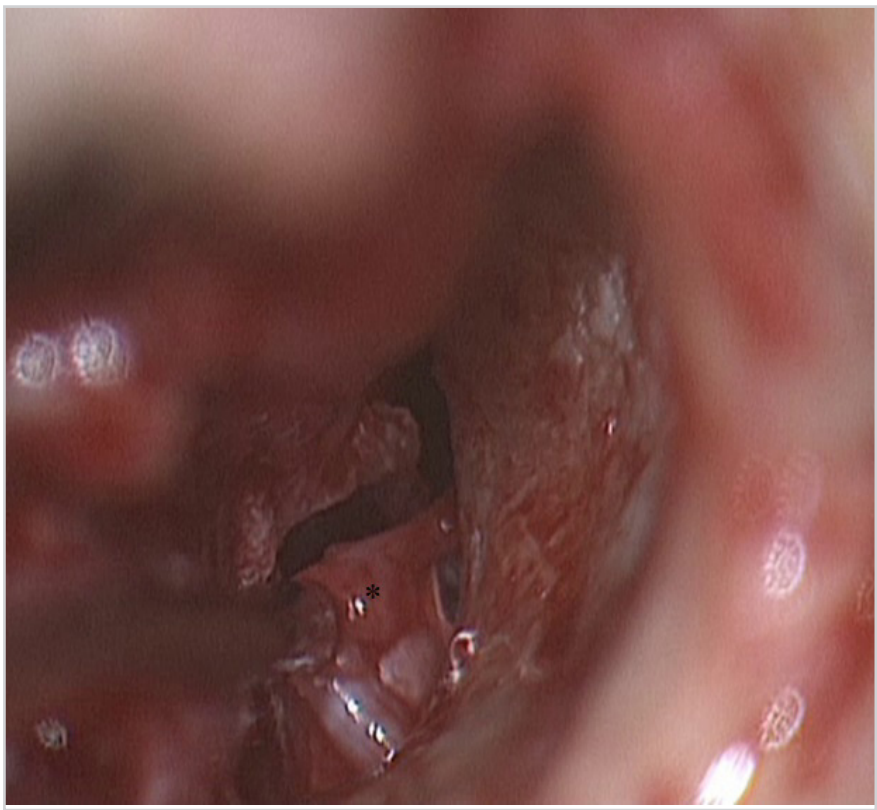

Figure 2. Intraoperative view of the thickened segment of the chorda tympani nerve at the tip of the suction tube (black asterisk) 


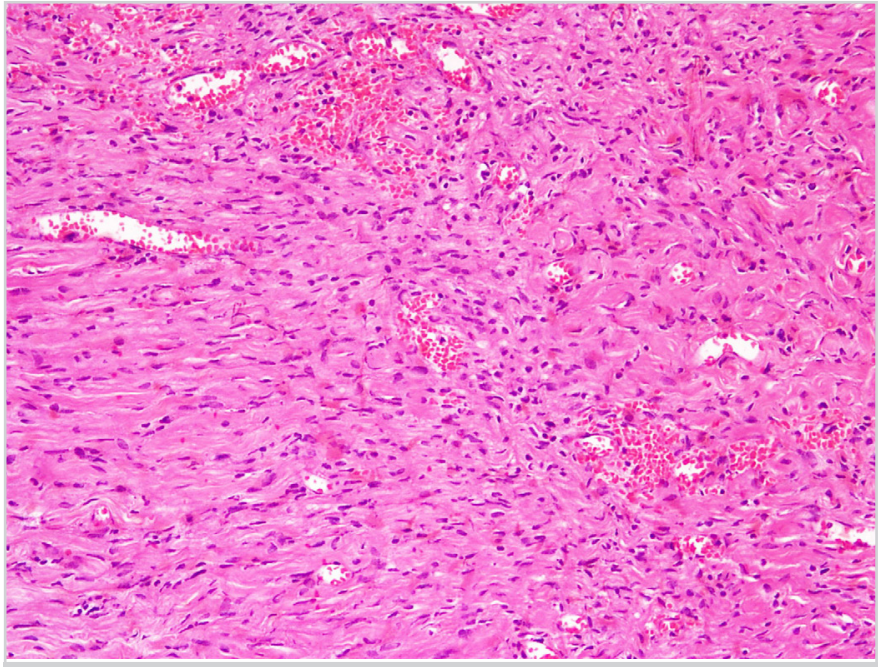

Figure 3. A histopathological section of the mesenchymal tumor is shown. The tumor is composed of uniform spindle cells accompanied by irregularly spaced vessels (x200, H\&E)

H\&E: Hematoxylin and eosin

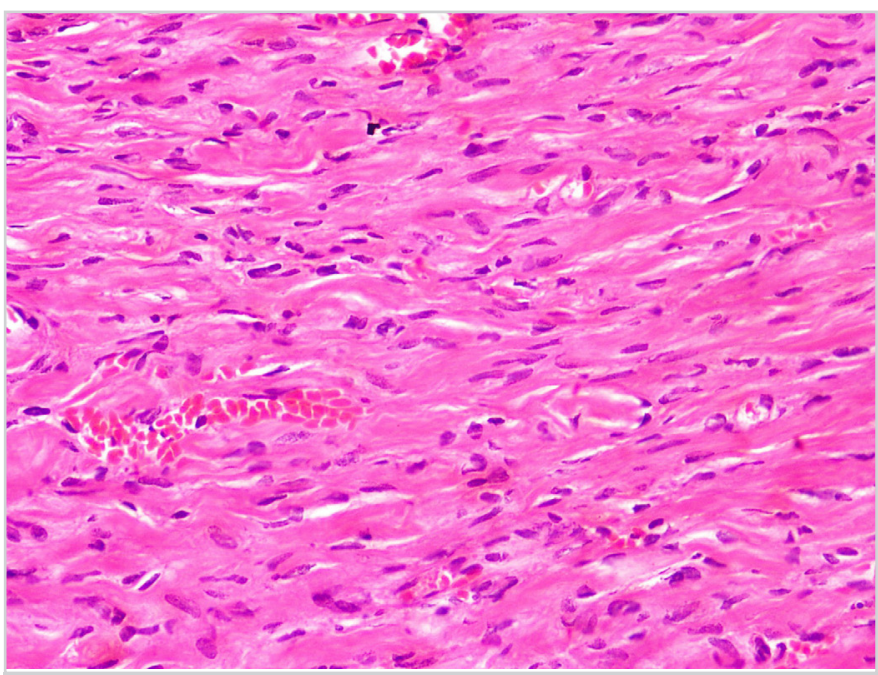

Figure 4. Narrow, elongated, and wavy cells with tapered ends are interspersed with collagen fibers (x400, $\mathrm{H} \& \mathrm{E})$

H\&E: Hematoxylin and eosin

perforation was closed, and there were no signs of ear discharge. The patient did not report any dysgeusia or hearing loss.

\section{Discussion}

Facial nerve schwannomas are benign, slow-growing tumors that destruct the surrounding structures. Expanding tumoral mass fills up the middle ear and the mastoid cavity and causes symptoms. They may be confused with cholesteatoma, paraganglioma, adenoma, meningioma, or other middle ear tumoral masses $(1,5)$. As the tumor size was very small in this case, our initial diagnosis was also chronic otitis media,

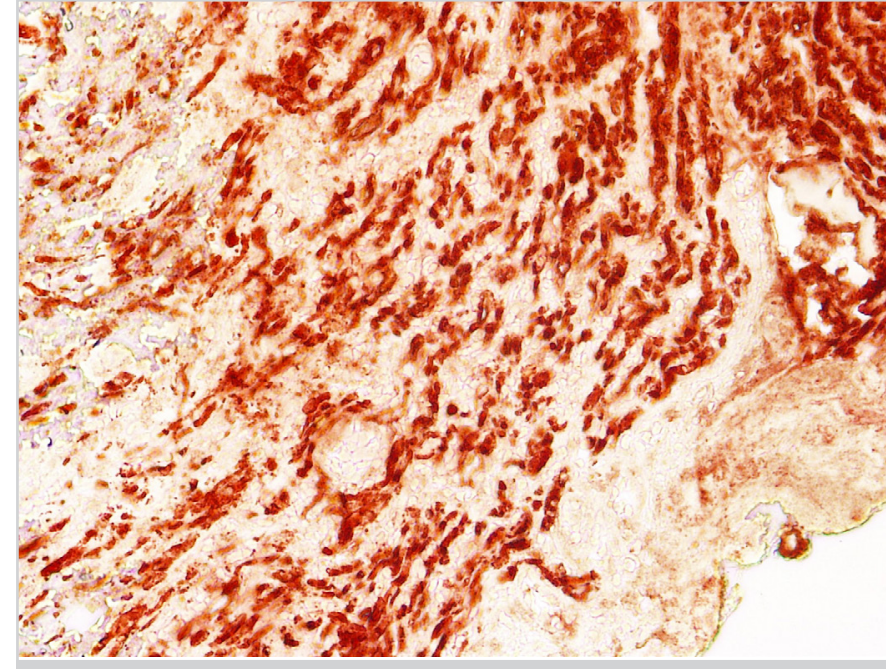

Figure 5. The tumor cells are stained positive for S100 (immunohistochemistry, $\mathrm{x} 400$ )

and we were not expecting to encounter any type of tumor in the middle ear.

Schwannomas may arise throughout the facial nerve or from the branches, such as chorda tympani and nerve to the stapedius muscles. CTS is quite rare. Patients present with slowly worsening conductive type hearing loss, tinnitus, and rarely facial weakness, or paralysis. Since facial paralysis is rarely appreciated in initial presentation and is mainly seen due to the mass pressure effect on the facial nerve itself; some authors advocate CTS as a distinct subgroup of facial nerve schwannomas $(6,7)$. Accordingly, asymptomatic CTS cases have been reported in the literature (2). Our patient had facial paralysis and purulent ear discharge in presentation, however, we believe such a small tumor was unlikely responsible for the facial paralysis. Due to the slow-growing nature of CTS and compensatory mechanisms, gustatory dysfunction has not been reported $(2,3,5)$. As in our case, gustatory dysfunction is rarely appreciated even after complete resection of the nerve $(2,5)$.

On otomicroscopic examination, a bulge on the posterosuperior quadrant of the tympanic membrane may be seen in CTS $(5,8)$. In our case, the presence of chronic otitis media concealed the diagnosis of middle ear mass, and there was no identifiable mass lesion on CT. Normally, osteolytic erosion of the ossicles and temporal bone with space-occupying soft tissue density along the course of the chorda tympani is observed in the CT scan $(6,7)$. Gadolinium-enhanced magnetic resonance imaging helps to better delineate the extent of the tumor and its relation with the facial nerve (8). Furukawa et al. (9) developed a new technique that combined $\mathrm{CT}$ and magnetic resonance imaging (MRI) images based on non-rigid registration and showed the benefits of this imaging in tumor localization 
and surgical planning. Both of the imaging techniques are complementary with each other and help the surgeon with preoperative planning $(6,10)$. As our initial diagnosis was chronic otitis media, we did not request MRI imaging.

Middle ear adenoma, paraganglioma, and meningioma are in the differential diagnosis of schwannoma during the histopathologic examination (8). Middle ear adenomas show variable patterns of glands/tubules composed of a uniform single layer of columnar cells with round hyperchromatic nuclei and eosinophilic cytoplasm. Immunohistochemically, these lesions are stained positively for pan-cytokeratin, and sometimes neuroendocrine differentiation can be seen. Paragangliomas have central, oval chief cells with a nested pattern, containing uniform nuclei with scattered chromatin and abundant eosinophilic granular cytoplasm. Chief cells are stained positively with chromogranin and synaptophysin. Sustentacular cells are stained positively with S100, which is difficult to see with hematoxylin-eosin staining. They settle at the periphery of the nests. Meningiomas show a whorls growth pattern, and they have oval or spindle cells with pale cytoplasm, intranuclear cytoplasmic inclusions, and indistinct cell borders. They may contain psammoma bodies. These tumors are EMA positive and S100 negative (3). In our case, the tumor cells were diffuse and strongly positive with S100; but negative for pan-cytokeratin, chromogranin, and synaptophysin.

Although there is no consensus about the best treatment strategy, restoration of hearing and maintaining normal facial motor functions are the mainstays of treatment for facial nerve schwannomas. In cases of facial paralysis due to the tumor itself, complete tumor excision with cable grafting is recommended (1). There is no need to restore the integrity of the chorda tympani nerve due to compensatory mechanisms. So, the tumor can be excised, or small asymptomatic tumors may be followed.

In the presented case, the facial paralysis was due to chronic otitis media, since CTS was very small in size, and not in the fallopian canal. Simple biopsying of the lesion was not possible. The small size of the tumor and the presence of chronic otitis media might complicate follow-up for this patient. Secondary surgeries would be required. So, we chose to excise the tumor completely.

\section{Conclusion}

Surgeons should be cautious about the underlying tumoral mass in cases of chronic otitis media and request imaging with contrast if suspected. Patients should be informed about the possibility of a middle ear tumor, therefore the decision to observe or excise can be made preoperatively.
Informed Consent: Informed consent was obtained

Peer-review: Externally peer-reviewed.

\section{Authorship Contributions}

Surgical and Medical Practices: K.D., M.Ö., B.Y.B., Ç.V., Concept: K.D., M.Ö., B.Y.B., Ç.V., Design: K.D., M.Ö., B.Y.B., Ç.V., Data Collection and/or Processing: K.D., M.Ö., B.Y.B., Ç.V., Literature Search: K.D., M.Ö., B.Y.B., Ç.V., Writing: K.D., M.Ö., B.Y.B., Ç.V.

Conflict of Interest: The authors declare that they have no conflict of interest to disclose.

Financial Disclosure: The authors declare that this study has not received any financial support.

\section{Main Points}

- Chorda tympani schwannomas are rare benign tumors of the middle ear cleft that may present with a bulge on the posterosuperior quadrant of the tympanic membrane.

- The otologic surgeons should be prepared for the incidental middle ear tumors which may also cause facial paralysis and chronic ear discharge.

- MRI with contrast and CT may be used together to better delineate the tumoral mass.

\section{References}

1. Dagli M, Salman N, Sancaktar ME, Kuran G, Guvey A, Yilmaz O. Facial nerve schwannoma located in the middle ear. J Contemp Med 2016; 6 (Case Reports): 72-5. [Crossref]

2. Ramírez-Camacho R, Trinidad A, Lois CG, Salas I, de Diego B, Roldán A, et al. Clinical and histological features of chorda tympani tumors. Int Adv Otol 2014; 10: 87-90. [Crossref]

3. Montava M, Giusiano S, Jolibert M, Lavieille JP. Chorda tympani schwannoma: one new case revealed during malignant otitis externa and review of the literature. Braz J Otorhinolaryngol 2018; 84: 252-6. [Crossref]

4. Schwam ZG, Kaul VZ, Wanna GB. Endoscopic resection of a chorda tympani schwannoma: A novel approach for an exceedingly rare entity. Laryngoscope 2019; 129: 1188-90. [Crossref]

5. Hopkins C, Chau H, McGilligan JA. Chorda tympani neuroma masquerading as cholesteatoma.J Laryngol Otol 2003; 117: 987-8. [Crossref]

6. Magliulo G, D’Amico R, Varacalli S, Ciniglio-Appiani G. Chorda tympani neuroma: diagnosis and management. Am J Otolaryngol 2000; 21: 65-8. [Crossref]

7. Kim CW, Han DH, Kim CH, Cho SJ, Rho YS. Primary middle ear schwannoma. Am J Otolaryngol 2007; 28: 342-6. [Crossref] 
Durmuş et al.

8. Undabeitia JI, Undabeitia J, Padilla L, Municio A. Chorda tympani neuroma. Acta Otorrinolaringol Esp 2014; 65: 263-5. [Crossref]

9. Furukawa T, Fabbris C, Ito T, Hiraka T, Goto T, Kubota T, et al. Facial nerve and chorda tympani schwannomas: Case series, and advantages of using non-rigid registration of post-enhanced 3D-T1 Turbo Field Echo and CT images (TURFECT) in their diagnosis and surgical treatment. Auris Nasus Larynx 2020; 47: 383-90. [Crossref]

10. Djerić D, Savić D. Otogenic facial paralysis. A histopathological study. Eur Arch Otorhinolaryngol 1990; 247: 143-6. [Crossref] 